\title{
Programação com blocos visuais para representar conceitos científicos por docentes da área de ciências
}

Marco Antonio Gomes Teixeira da Silva - PPGCN, CCT, UENF - marcoagts@gmail.com Sergio Luiz Cardoso - CCT, UENF - sergio.luis64@gmail.com

Resumo: Este artigo é parte de uma pesquisa de mestrado e tem por objetivo identificar como a tecnologia pode proporcionar habilidades para o desenvolvimento de Objetos Digitais de Aprendizagem (ODA) para representação de conceitos científicos, empregando programação com blocos visuais, na formação inicial e continuada do docente. Durante um curso de capacitação foram desenvolvidos ODA e, após, avaliados, por professores convidados, aplicando a escala de Likert. Considerando os dados obtidos e o material utilizado, é razoável assumir que a presença das tecnologias digitais na formação docente pode proporcionar habilidades e competências que permitam aos docentes desenvolver ODA com adequação aos conceitos científicos. Também é possível ponderar a viabilidade de capacitá-los com programação em blocos visuais.

Palavras-chave: Objetos Digitais de Aprendizagem. Capacitação. Formação docente.

\section{Programming with visual blocks to represent scientific concepts per science area teachers}

Abstract: This article is part of a master's research and aims to identify how technology can provide skills for the development of Digital Learning Objects (DLO) for the representation of scientific concepts, using visual block programming, in initial and continuing education of the teacher. During a capacity build course, DLO were developed and after then evaluated by invited teachers using the Likert scale. Considering the data obtained and the material used, it is reasonable to assume that the presence of digital technologies in teacher education can provide skills and competences that enable teachers to develop DLO that is adapted to scientific concepts. It is also possible to consider the feasibility of enabling them with programming in visual blocks.

Keywords: Digital Learning Objects. Capacity build. Teacher education.

\section{Introdução}

Os recursos das Tecnologias Digitais da Informação e Comunicação (TDIC) entusiasmam transformações na educação (Valente, 2016), entretanto, demandam conhecimento inicial e atualização constante. Entre as possibilidades encontradas nas TDIC, que podem proporcionar a criação de diferentes recursos para áreas distintas, destaca-se a programação.

Apesar de ser uma aplicação específica da área de engenharia, historicamente, segundo Valente (2016) a programação se destaca nas atividades de informática na educação. Para o autor, esse processo foi possível, inicialmente pela linguagem "Logo", criada por Seymour Papert na década de 1960.

Valente, já em 1999 (p. 13) afirmava que as licenciaturas deveriam "criar condições para que o participante do curso construa conhecimento sobre as técnicas computacionais".

O licenciando que desenvolve habilidades computacionais possui um facilitador para aliar tecnologia, com todos os seus recursos, à prática pedagógica (Basniak; Silva, 2018; Valente, 1999). Nesse sentido a deficiência de determinadas habilidades relativa as V. $17 \mathrm{~N}^{\circ}$ 3, dezembro, 2019 RENOTE DOI: 
das TDIC desfavorece o enriquecimento tecnológico do professor e, consequentemente, a apropriação de práticas inovadoras em suas práxis.

É indispensável considerar o formato dos cursos para que o docente aproprie-se do aprendizado para fazer a conexão das TDIC na sua prática. Pois, quando as TDIC estão presentes na formação, em geral, prioriza as técnicas em si e, não as aplicações dos recursos para o ensino na área de formação. Um exemplo são os cursos de programação, que se preocupam com a sintaxe e não a assimilação da técnica para a aplicação na educação (Dasgupta; Resnick, 2014).

Outro ponto que favorece a área de programação, é apontado por Santos e Bezerra (2017), os quais afirmam que este recurso promove a formação do pensamento computacional, apresentando-se como uma habilidade cognitiva que ultrapassa a destreza de usar o computador e a rede de internet. Apesar desta constatação os autores apontam que no Brasil, essa área ainda não está de forma clara na Base Nacional Comum Curricular (BNCC) da educação básica, distanciando-se do processo emergente que surge na Europa.

Ao buscar no documento da $3^{\text {a }}$ versão da BNCC (Brasil, 2017), a expressão "pensamento computacional", é relacionada na etapa do ensino fundamental com a área de matemática e, somente na etapa do ensino médio, aparece a relação do "pensamento computacional" associado as aplicações das TDIC, além de, também, ser promovida pela pela $7^{\mathrm{a}}$ competência específica (Mobilizar práticas de linguagem no universo digital).

Valente (2016) também contribuiu definindo que a programação associa estratégias: ascendente, heurística, pensamento divergente, criatividade, resolução de problemas, pensamento abstrato, interação, recursividade, métodos colaborativos e metacognição. Autores, como Santos e Bezerra (2017), Valente (2016) e Wing (2006), incluem essas relações como o "pensamento computacional".

Apesar das constatações acima, que chamam atenção para a questão da programação, na formação do docente, neste estudo, busca-se identificar: Como a programação pode proporcionar habilidades para o desenvolvimento de objetos de aprendizagem de conceitos nas áreas de ciências naturais e matemática, considerando a formação inicial e/ou continuada do docente?

$\mathrm{Na}$ investigação proposta, definiu-se como objetivos: analisar a viabilidade de capacitar docente na formação inicial e continuada, empregando programação em blocos visuais; e, avaliar as propostas de representação conceitual em relação as adequações aos conceitos formalizados nos recursos desenvolvidos pelos participantes da pesquisa, nas áreas de ciências naturais e matemática.

Este estudo observa que as Tecnologias da Informação e Comunicação (TIC) sempre estiveram presente na atividade docente, sejam por intermédio de quadros, mapas, imagens de tabelas e outros recursos, conforme cita Gabriel (2013). Assim sendo, nesta pesquisa, as TDIC na educação, é um "recorte" das TIC, bem como, também delimita-se pelas as áreas da biologia, física, química e matemática, as quais serão definidas no decorrer do texto como ciências.

A seguir, no texto, é tratado sobre o embasamento de softwares educacionais, com a finalidade de identificar os dados coletados; apresentado o método empregado para coletar e analisar os dados; exposto os dados coletados; discussão e consideração sobre a pesquisa e os objetivos.

\section{Softwares e aplicativos educacionais}

O ambiente de programação em blocos e seus possíveis Objetos de Aprendizagem (OA) desenvolvidos, com objetivo educacional, podem ser considerados como softwares e/ou aplicativos de uso pedagógico. A utilização dos OA tem por finalidade promover e 
facilitar o processo de aprendizagem. Os Softwares e Aplicativos Educacionais (SeAE) tem como diferencial dos demais (de uso comercial ou das engenharias), que devem ser desenvolvidos tendo como base uma concepção pedagógica. Portanto, devem empregar imagens, sons e lógicas que atraíam a atenção do usuário, com a finalidade de construir ou introduzir um conceito científico (Gouvêa; Nakamoto, 2015).

Os SeAE podem ser classificados como: (i) Tutoriais (programas nos quais a informação é organizada de acordo com uma sequência pedagógica e apresentada ao aluno nessa ordem); (ii) Simulação e modelagem (simulam fenômenos reais no ambiente digital, permitindo uma interação maior com usuário); (iii) Jogos (têm por objetivo desafiar e motivar o usuário por meio de competição com o computador ou com outros adversários e geralmente possuem estratégia, estética, recursos de multimídia e uma história); (iv) Programação (os usuários utilizam-se de conceitos, estratégias e capacidade de resolver problemas) (Gouvêa; Nakamoto, 2015; Valente, 1999). Existem outras classificações como processador de texto e hipertexto, por exemplo, no entanto os citados atendem às perspectivas desta pesquisa.

Para Valente (1999) os recursos da área de computação na educação consideram dois paradigmas: construcionista e instrucionista. O uso do recurso digital para representação dos métodos tradicionais de ensino é o paradigma instrucionista (tutoriais, prática, modelagem, jogos e simulação fechados). Nessa prática o computador é utilizado para fazer perguntas e obter respostas, com pouca interação entre o estudante e o SeAE. Já o paradigma construcionista (programação, simulação aberta e jogos de estratégias organizadas para tal) compreende a "construção" pessoal do conhecimento, dentro do recurso proposto, em que se observa uma interação do usuário, onde a reflexão do processo define-se em uma depuração do conceito e das estratégias aplicadas.

Valente (2014) argumenta que o uso de SeAE, facilitam a "construção" do conhecimento. Para o autor a possibilidade de animar objetos na tela do computador é uma ferramenta relevante, principalmente nas áreas de ciências.

O recurso de programação com blocos visuais, ao mesmo tempo que viabiliza desenvolver OA no formato digital, em que a própria interface de programação também é um recurso das TDIC para o processo de ensino/aprendizagem, pois, permite criar competências digitais e o pensamento computacional (Santos; Bezerra, 2017; Valente, 2016).

Segundo Dasgupta e Resnick (2014), o ambiente de programação com blocos visuais, é uma solução para a carência de habilidades deste recurso e da excessiva ênfase que é dada a técnica, que permite desenvolver simulações, integrando diferentes mídias (sons, imagens, texto).

Neste estudo é considerado o termo “Objeto Digital de Aprendizagem” (ODA), para os recursos desenvolvidos pelos participantes, pois serão os aplicativos construídos na interface de programação em blocos visuais. Assim, como também, adota-se a definição para ODA encontrada em Arrais (2016): recurso com planejamento pedagógico, com conteúdo organizado para atender a um conceito científico, interativo e desenvolvido em ambiente digital.

\section{Metodologia}

Esta investigação foi realizada durante a aplicação de um curso de extensão, desenvolvido com o objetivo de fornecer aos participantes competência com programação em blocos visuais para posterior construção de ODA, com a interface de programação Scratch 2.0. A elaboração do ODA foi condição para receber o certificado de participação e conclusão.

O curso transcorreu entre março e junho de 2018 e teve a duração de 120 dias. Foi organizado e desenvolvido em um Ambiente Virtual de Educação e Aprendizagem 
(AVEA) - na plataforma Moodle -, disponível para acesso remoto em servidor da Universidade Estadual do Norte Fluminense Darcy Ribeiro (UENF) e foi ofertado para voluntários docentes formados e licenciandos da área de ciências, os quais concordaram em participar da pesquisa.

Durante o curso foram adotadas estratégias que envolviam a capacidade do participante para resolver problemas propostos, empregando a metodologia construcionista. As etapas do curso no AVEA foram organizadas em tópicos e mediadas pelos pesquisadores, para os participantes conhecerem a interface de programação, construírem pequenos projetos para aprendizado e o projeto final.

Após as etapas do curso, foi discutido em um fórum sobre o uso de material digital com emprego pedagógico. Nessa fase o objetivo era despertar no participante a sua formação pedagógica, para construção do ODA. No fórum, foi apresentado um pequeno texto sobre o uso de recurso digital em sala, como material pedagógico de apoio e, solicitado que participantes iniciassem o planejamento do ODA.

Os objetos foram desenvolvidos individualmente pelos participantes, em um período de 30 dias, após as etapas no AVEA. Esses ODA foram avaliados por meio de questionários, enviados por e-mail para diversos docentes não participantes do curso, das respectivas áreas dos ODA confeccionados. No questionário enviado aos convidados é relatada uma definição sumária da pesquisa e solicitada autorização para publicar os dados fornecidos.

A disposição dos itens no questionário utilizado na investigação, foi organizado em quatro dimensões: (i) Pedagógica, que observou a linguagem empregada, o tipo de aprendizagem e associação do ODA com conhecimentos prévios para indicar se o material pode ser classificado como potencialmente significativo e a interdisciplinaridade do recurso com a área de ciências; (ii) Técnica, que buscou informações sobre a organização dos objetos, sons, cores, animações e se o contexto organizacional estava legível; (iii) Conceitual, a qual é a maior dimensão, uma vez que, um dos objetivos era a relação do uso das TDIC com a viabilidade conceitual científica. Nessa dimensão, foi levantada não só a relação conceitual, mas também, o formato empregado e a questão do simbolismo; e, (iv) Pessoal, a qual abordou se o avaliador identifica o ODA como meio auxiliar para o processo de ensino, qual etapa e o formato que o autor do objeto utilizou. Nessa fase da investigação, o avaliador poderia ainda dar sua opinião livre em cada ODA.

A análise dos dados foi realizada em dois grupos: docentes formados e graduandos. Foi considerado a média aritmética de cada ODA, organizados por área, nos respectivos grupos. A apreciação dos dados foi desenvolvida por meio de análise dos gráficos construídos com base na média aritmética das áreas de cada grupo (docentes e graduandos).

\section{Resultados e discussão}

Do universo de 62 participantes que se cadastraram inicialmente o para o curso, concluíram a capacitação, com a entrega dos ODA, 18 participantes, representando $29,03 \%$ do total inicial. Sendo considerado para esta análise apenas os 18 participantes: $10(55,60 \%)$ alunos em formação (nove de ciências e um bacharelando em engenharia de software) e 8 $(44,40 \%)$ docentes formados (sete de ciências e um da área de letras) (Figura 1).

$\mathrm{Na}$ figura 1 observa-se que os participantes da área de química predominam entre os "licenciandos" e, entre os docentes, destaca-se a área de matemática, seguida pela de química. Ao notar à heterogeneidade dos participantes concludentes, observa-se a formação dos docentes em: (i) três com pós-graduação lato sensu (especialistas) e (ii) quatro com formação stricto sensu (um doutor e três mestres). 


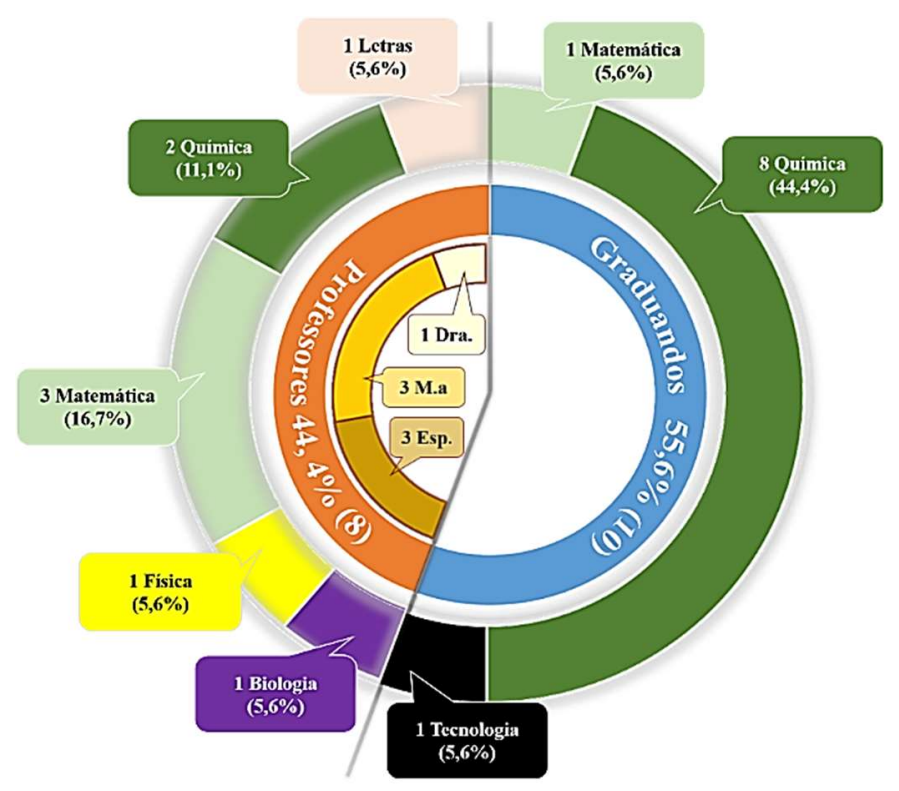

Figura 1 - Gráfico dos números de participantes que concluíram o curso com certificação

Os ODA desenvolvidos pelos participantes foram classificados em: tutoriais; jogos; exercício e prática; simuladores; e, modelagem (Quadro 1), empregando a definição dada por Gouvêa e Nakamoto (2015) e Valente (1999).

\begin{tabular}{|c|c|c|c|c|}
\hline Áreas & Biologia & Física & Matemática & Química \\
\hline Docente & 1 Jogo; & $\begin{array}{l}1 \text { Tutorial; } \\
1 \text { Simulador / } \\
\text { Tutorial; }\end{array}$ & $\begin{array}{l}1 \text { Jogo / Exercício e prática; } \\
1 \text { Modelagem / Exercício e } \\
\text { prática; }\end{array}$ & $\begin{array}{l}1 \text { Tutorial / } \\
\text { Exercício e prática; } \\
2 \text { Tutoriais; }\end{array}$ \\
\hline Graduando & & $\begin{array}{l}1 \text { Simulador / } \\
\text { Exercício e } \\
\text { prática; }\end{array}$ & $\begin{array}{l}1 \text { Tutorial / Exercício e } \\
\text { prática; }\end{array}$ & $\begin{array}{l}7 \text { Simuladores; } \\
1 \text { Tutorial / } \\
\text { Exercício e prática }\end{array}$ \\
\hline Total & 1 Jogo & $\begin{array}{l}1 \text { Tutorial } \\
2 \text { Simuladores* }\end{array}$ & $\begin{array}{l}1 \text { Modelagem } \\
1 \text { Jogo } \\
1 \text { Tutorial }\end{array}$ & $\begin{array}{l}4 \text { Tutoriais } \\
7 \text { Simuladores }\end{array}$ \\
\hline
\end{tabular}

Nota: $(*)$ um objeto foi classificado com o formato simulador e tutorial.

Fonte: Dados da pesquisa - etapa de análise dos ODA.

É relevante salientar que os professores formados, os quais possuem um maior tempo de desenvolvimento com a prática pedagógica, tenderam para os jogos, tutoriais e exercícios, já os graduandos tiveram maior tendência para a simulação, considerando a classificação apresentada neste estudo.

\subsection{Análise das avaliações}

Após avaliação dos ODA realizada pelos professores convidados, os dados foram tabulados e organizados com base na média entre os grupos de docentes e graduandos por área. Nessa fase observou-se algumas divergências entre esses os dois grupos (Figura 2). $\mathrm{Na}$ análise do gráfico buscou-se identificar os vales na curva que representava os dados avaliados dos docentes na dimensão pedagógica e conceitual, pois apresentavam-se mais acentuados que a linha dos graduandos. 


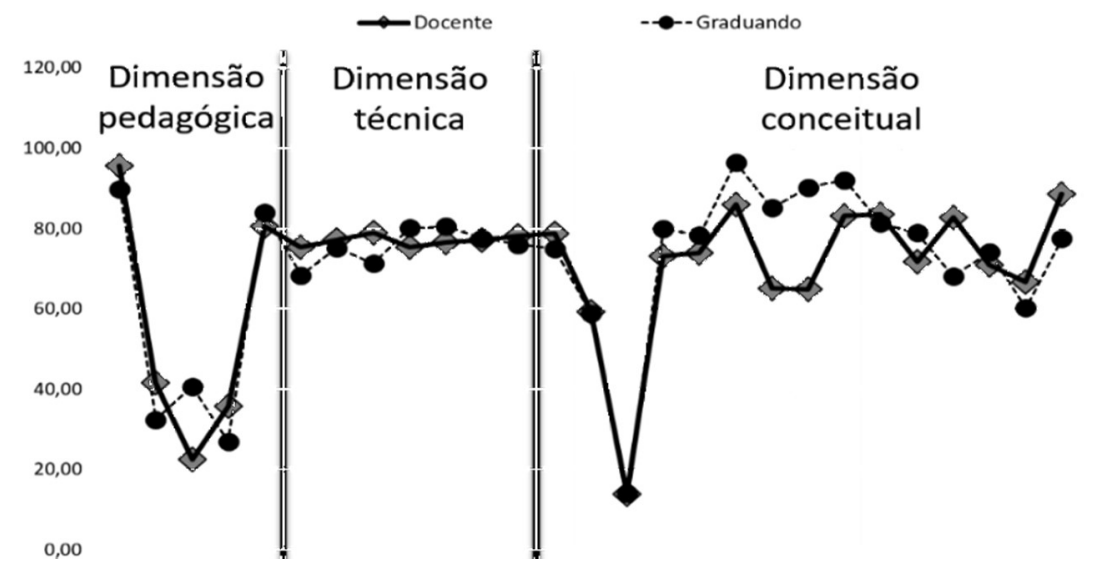

Figura 2 - Gráfico comparativo das médias dos dados entre docente e graduando

Com base na média de cada área (biologia, física, matemática e química) identificou-se que a linha correspondente ao grupo docentes, da área de biologia (B01), tendeu para um vale.

Pelo exposto no parágrafo anterior, para identificar a relação das três dimensões principais analisadas (pedagógica, técnica e conceitual), foi reconstruído o gráfico, excluindo os valores da avaliação do recurso B01. No gráfico da Figura 3, observa-se que, entre docente e graduando, as linhas que representam a média das avaliações ficaram próximas. Confirmando a tendência relatada pela literatura, em que o aluno tende a repetir o que aprende em sala com o docente (Basniak; Silva, 2018; Valente, 1999 e 2016).

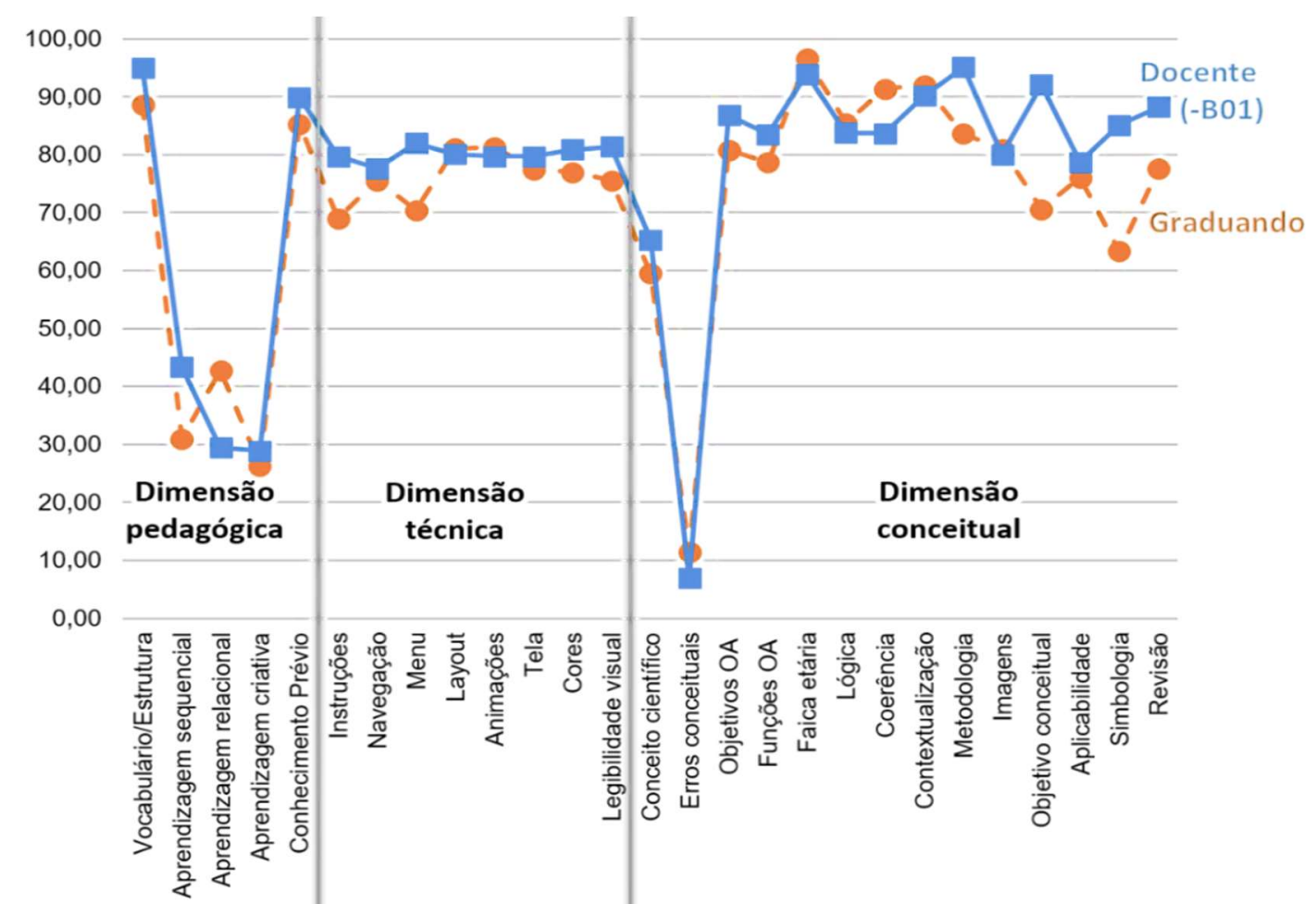

Figura 3 - Gráfico comparativo das médias dos dados entre docente (excluído o ODA B01) e graduando

Na figura 3, em que é exibido a média da avaliação dos ODA, alguns itens das três dimensões apresentam uma leve variação, indicando uma maior aceitabilidade nos ODA desenvolvidos pelos docentes. Contudo, a alteração é leve, sendo identificável devido o gráfico conter apenas as duas linhas comparativas (docentes e graduando). 
A dimensão pedagógica apresentou uma diferença, onde os graduandos tiveram maior influência pelo emprego de uma aprendizagem relacional. Aprendizagem essa que identifica as relações com outros fatos ou outras fontes de informação e promove interações, ou seja, está incluso no processo da aprendizagem significativa. Já os docentes, apresentaram maior aderência pela aprendizagem sequencial, na qual $o$ conteúdo é passado e memorizado, para o usuário repetir quando for solicitado.

A área aprendizagem criativa, foi avaliada de forma equilibrada para ambos os grupos. $\mathrm{Na}$ aprendizagem criativa ocorrem possibilidades de interação entre pessoas e tecnologias, existe compartilhamento de objetivos comuns, desenvolvimento de atividades com interação do usuário de forma ampla.

$\mathrm{Na}$ dimensão técnica, os grupos ficam muito próximos, com diferença no item das informações e instruções apresentadas na tela e menu. Sendo esses itens desfavorecendo aos graduandos.

$\mathrm{Na}$ dimensão conceitual, os graduandos obtiveram uma avaliação abaixo dos docentes, nos itens sobre metodologia, adequação do ODA ao objetivo conceitual proposto, simbologia adotada e revisão. Também, observa-se que a linha pontilhada (dos docentes, excluindo o B01), tendeu para cima nestes mesmos itens.

\subsection{Discussão}

É relevante recordar que o grupo amostral participante da pesquisa é organizado em dois: docentes formados e graduandos. Desses grupos, destaca-se que do universo inicial da pesquisa (62) no cadastramento, apenas quatro participantes informaram ter conhecimentos teóricos sobre a área de programação. Também, ressalta-se que os participantes, em interações pontuais na plataforma AVEA do curso, definiram a área de programação em blocos visuais como viável pedagogicamente.

Nesse sentido, a área de programação em blocos, como disciplina e/ou curso de extensão, pode ser refletida para adquirir o pensamento computacional, exigindo do indivíduo que se aventura nesse processo conhecimentos de: estratégia, heurística, pensamento divergente, criatividade, resolução de problema, abstração, recursividade e metacognição, conforme definiu Jeannettte Wing (2006). Assim, além de ser uma área que possibilita ao docente representar os conceitos científicos e não apenas transitar por eles em uma explanação teórica, desenvolve no docente ações que contribuem para suas atividades de forma lógica e eficiência na resolução de problemas do mundo real.

Os dados obtidos indicam que esse conhecimento pode ser um facilitador, apesar disso, deve-se considerar a escolha da ferramenta e forma de abordagem coerente para viabilizar o processo, conforme citaram Dasgupta, Resnick (2014) e Valente (2016) e, ainda, para que o processo venha oportunizar a formação da competência digital, como foi apontado por Redecker (2017).

Ao desenvolver os ODA os participantes permitiram uma análise diferenciada. Inicialmente, é preciso considerar que entre o grupo de docentes e dos futuros docentes não há uma diferença que se defina como "alarmante", pois, ambos participaram do curso de extensão e, logicamente obtiveram a mesma formação para atender esta análise. Nesse aspecto, na Figura 3 foi demonstrado que na dimensão técnica não houve muita divergência, mas duas leves variações entre instruções na tela e menu, sendo favorável aos docentes.

$\mathrm{Na}$ análise fica evidenciada a relevância das TDIC, "recortado" na área de programação, conforme identificou a análise dos ODA desenvolvidos pelos participantes e avaliados por professores convidados.

O número de tutoriais se explica pelo fato do docente ter formação nos sistemas apostilados e cópias de textos, conforme aponta Rodrigues, De Almeida e Valente (2017). 
Contudo, se constata o formato de ODA como tutoriais em ambos os grupos, não apenas nos docentes, que são considerados os imigrantes digitais.

Então, destacando novamente que a questão principal dessa pesquisa é sobre a contribuição que as TDIC ofereceriam no processo de representação do conceito científico, considerando a programação em blocos visuais. Inicialmente, é de se esperar que o docente com maior prática, também, tenha maior domínio dos conceitos científicos. Atentando para a proposta desta investigação e os dados obtidos, os docentes e graduandos conseguiram desenvolver processos e representar o conceito científico empregando a programação em blocos visuais.

A averiguação dos objetos na dimensão conceitual ocorre uma variação, mais acentuada que as demais dimensões. Os itens metodologia e adequação do ODA ao objetivo conceitual destacam-se pela linha que representa a média das avalições dos docentes ao fazer pico e a média das avaliações dos graduandos criam um vale. Ou seja, nesses dados, o docente em sala possui uma visão melhor da metodologia e do objetivo. Tal fato é possível identificar na literatura de Valente $(2016 ; 1999)$. Ao mesmo tempo vai de acordo com Demo (2009) que afirma que o docente após formado aprende, pesquisando para ensinar.

De forma sucinta, na análise dos dados das três dimensões (pedagógica, técnica e conceitual), pode-se dizer que não houve divergência preocupante, apenas identificação do que é apontado na literatura.

Na dimensão pessoal da análise dos ODA foram realizadas críticas, pelos avaliadores convidados, os quais definiram em geral que os recursos são viáveis para uma revisão de conteúdo ou introdução. Uma apreciação comum, identificada pelos professores convidados, foi a falta de exploração do recurso para que se tornasse mais interativo. Observando os dados contidos no Quadro 1, essa crítica apontada é clara, pois foram desenvolvidos ODA com formato tutoriais e esse processo não cria interações com usuário.

Algumas críticas pontuais surgiram para os dois grupos: falta de recursos inclusivos ou problemas de cores para alguns transtornos e manipulação não intuitiva.

Em relação ao objeto $\mathrm{B} 01$, é necessário considerar que o desenvolvedor se preocupou na etapa de programação do projeto de um jogo com fases e, tal processo, tendo em vista o pouco contato com a ferramenta, pode ter inviabilizado a abordagem dos itens da dimensão conceitual. Ou seja, esse participante se preocupou com a implementação da técnica, tentando desenvolver um projeto de maior aspecto técnico que o prazo permitia e, por consequência, não atentou para o principal fator analisado nessa pesquisa: o emprego do recurso para facilitar o entendimento do conceito científico conforme menciona Dasgupta e Resnick (2014).

Ao observar a produção dos ODA dos professores e graduandos separadamente, nota-se que os licenciandos demonstram-se influenciados pelo uso das TDIC com formato mais interativo (simulação) e os docentes se afirmam em uso de materiais digitais para expor o conteúdo, como os tutoriais.

\section{Conclusão}

Esta pesquisa identificou, por meio da metodologia adotada, que o docente, seja ele formado ou em formação, é capaz de utilizar a programação em blocos para representar o conceito científico. Assim, poderá o próprio se tornar autor de seu material. Portanto, considera-se que as TDIC, no que diz respeito à área de programação com blocos visuais, devem ser apropriadas pelos docentes desde a formação inicial. Ressaltase, que tanto na formação inicial quanto na continuada, para que as TDIC proporcionem habilidades e competências digitais e os docentes possam desenvolver seus materiais, é necessário que as capacitações e habilitações ocorram com relação à sua respectiva 
formação pedagógica.

Os dados obtidos, dentro do grupo amostral selecionado, permitiram observar que a formação inicial docente é uma etapa importante para que seja desenvolvida a competência digital, considerando a área de programação. Todavia, a formação continuada é uma ferramenta que sustenta a atualização do docente. Ponderando que a tecnologia não é estanque, muito ao contrário disso, a tecnologia se assegura a cada dia, dentro de inovações, conforme comenta Valente (2016).

Portanto, também se considera que a questão de pesquisa e o primeiro objetivo específico, foi alcançado, pois, os participantes identificaram a viabilidade do material e do formato empregado, para capacitar docentes e graduandos na utilização de programação em blocos. Sinalizando que o ambiente de programação empregado neste estudo é também um recurso viável para a sala de aula.

Já o segundo objetivo, a avalição dos ODA, buscou identificar a viabilidade, considerando que no estudo desenvolvido as linhas do gráfico (Figura 3) das avaliações dos ODA ficaram acima do $60 \%$ de aceitação dos avaliadores convidados, tanto para docentes, quanto para graduandos. Nos gráficos analisados só ocorreram vales na dimensão pedagógica (pois os dados obtidos foram organizados em três partes dentro do mesmo número de participantes dos demais itens) e nos erros cometidos, os quais ficaram abaixo de $10 \%$.

Quanto ao formato para desenvolver a proficiência computacional no docente, é importante identificar que essa área é dinâmica e, isso a torna complexa. De tal modo, deve-se conceber recursos de capacitação com base na área de formação pedagógica do docente. Tal relação diminui a insegurança para aprender o "novo" recurso tecnológico.

Por fim, é coerente identificar que a tecnologia não é a solução, mesmo porque é importante saber: solução para quê? Mas, identificar que a tecnologia pode ser um recurso de apoio metodológico e não considerar que aulas expositivas em slides são uso de tecnologias ativas para o aprendizado, como apontaram Basniak e Silva (2018), Martins e Giraffa (2016) e Valente (2014). E, apesar das considerações deste estudo apontarem bons indicadores, também expõem que é necessário investigar o formato que abordagem das TDIC na formação inicial.

AGRADECIMENTO: O presente trabalho foi realizado com apoio da Coordenação de Aperfeiçoamento de Pessoal de Nível Superior Brasil (CAPES) - Código de Financiamento 001; e, do PPGCN da Universidade Estadual do Norte Fluminense Darcy Ribeiro.

\section{Referências Bibliográficas}

ARRAIS, M. Objetos de Aprendizagem: conceito e estrutura básica. Linha direta, p. 14-16, mar. 2016.

BASNIAK, M. I.; SILVA, S. DE C. R. Tecnologia em processos culturais de ensino revelados por professores. Revista de Ensino de Ciências e Matemática, v. 9, n. 4, p. 169-182, 2018.

BRASIL. Resolução CNE/CP 2/2017. Institui e orienta a implantação da Base Nacional Comum Curricular. Diário Oficial da União: Seção 1, Brasília, DF. 22 de dezembro de 2017, Seção 1, pp. 41 a 44. 
DASGUPTA, S.; RESNICK, M. Engaging novices in programming, experimenting, and learning with data. ACM Inroads, v. 5, n. 4, p. 72-75, 2014.

DEMO, P. Educação hoje: "novas" tecnologias, pressões, e oportuidades. São Paulo: Atlas, 2009.

GABRIEL, M. Educar. São Paulo: Saraiva, 2013.

GOUVÊA, M. C. M. DE; NAKAMOTO, P. T. Avaliação de software educacional: uma oportunidade de reflexão da educação na sociedade do conhecimento. In: ENCONTRO DE PESQUISA EM EDUCAÇÃO, 8., 2015. Anais [...] Uberaba: Universidade de Uberaba, 2015.

MARTINS, C.; GIRAFFA, L. M. M. Design de práticas pedagógicas incluindo elementos de jogos digitais em atividades gamificadas: Design of educational practices, including digital game elements applied to gamified activities. Obra digital, n. 10, p. 52-67, 2016.

REDECKER, C. European Framework for the Digital Competence of Educators: DigCompEdu. Seville: Joint Research Centre, 2017.

RODRIGUES, A.; DE ALMEIDA, M. E. B.; VALENTE, J. A. Currículo, narrativas digitais e formação de professores: Experiências da pós-graduação à escola. Revista Portuguesa de Educação, v. 30, n. 1, p. 61-83, 2017.

SANTOS, G. P. DOS; BEZERRA, R. DOS S. Desenvolvendo o pensamento computacional utilizando Scratch e lógica matemática. In: JORNADA DE ATUALIZAÇÃO EM INFORMÁTICA NA EDUCAÇÃO, 6., 2017. Anais [...]. Recife, PE: Sociedade Brasileira de Computação, 2017, p. 66-99. Disponível em: http://www.br-ie.org/pub/index.php/pie/article/view/7214. Acesso em 16 fev. 2019.

VALENTE, J. Integração do pensamento computacional no currículo da educação básica: Diferentes estratégias usadas e questões de formação de professores e avaliação do aluno. Revista e-Curriculum, v. 14, n. 3, p. 864-897, 2016.

VALENTE, J. A. O computador na sociedade do conhecimento. Campinas: NIED/UNICAMP, 1999.

VALENTE, J. A. A Comunicação e a Educação baseada no uso das Tecnologias Digitais de Informação e Comunicação. UNIFESO - Humanas e Sociais, v. 1, n. 01, p. 141-166, 2014.

WING, J. M. Computational Thinking. Magazine Communications of the ACM, v. 49, n. 3, p. 33-35. DOI: 10.1145/1118178.1118215. 